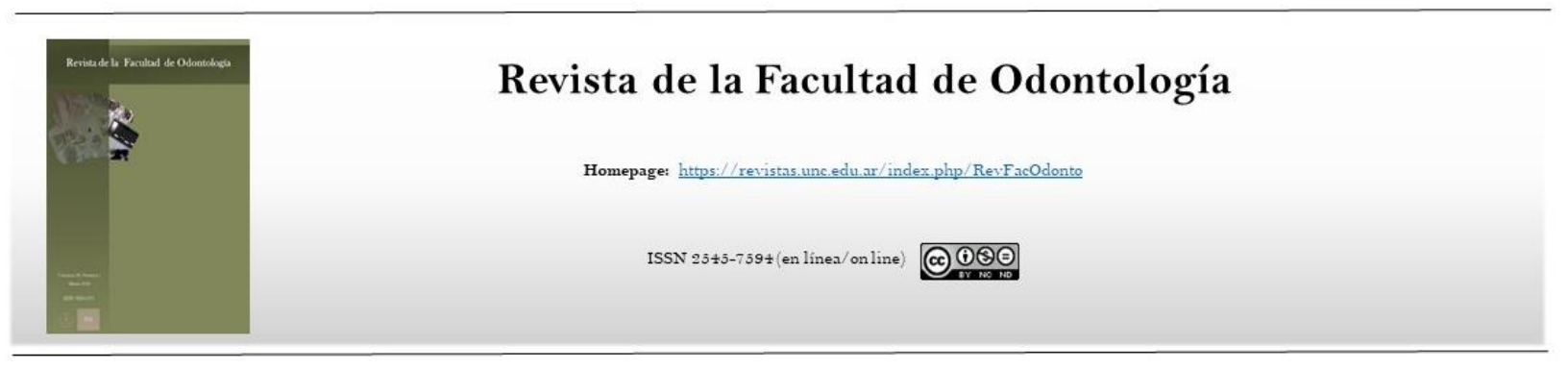

Adenoma pleomórfico de glándula lagrimal: localización atípica de un tumor salival

\title{
Pleomorphic adenoma of the lacrimal gland: atypical location of a salivary tumor
}

\author{
Gómez Rosso María A , Samar María E ${ }^{1}$, Ávila Rodolfo E² Ferraris Luis $^{3}$, Fonseca Ismael ${ }^{4}$, Fernández Javier E ${ }^{1}$ \\ ${ }^{1}$ Departamento de Biología Bucal. Facultad de Odontología. Universidad Nacional de Córdoba. samarcongreso@gmail.com \\ ${ }^{2}$ Cátedra de Biología Celular, Histología y Embriología. Facultad de Ciencias Médicas. Universidad Nacional de Córdoba \\ ${ }^{3}$ Servicio de Patología. Hospital San Roque. Córdoba. \\ ${ }^{4}$ Cátedra II de Patología. Facultad de Ciencias Médicas. Universidad Nacional de Córdoba
}

\begin{abstract}
Introduction: In daily ophthalmological practice, lacrimal gland tumors are rare. They represent 5 to $7.5 \%$ of all intraorbital tumors. The most common epithelial tumor of this gland is the pleomorphic adenoma, with a percentage of 25 to $50 \%$ of its tumor lesions. Objective: In this communication we presented a case of lacrimal gland pleomorphic adenoma and study the expression of Ki67 and the location and expression of MUC-1 and its correlation with tumor prognosis. We also carried out a retrospective descriptive study of the literature on the subject published between 1951 and 2020, using the MEDLINE database. Material and methods: The surgical piece examined, was processed according to the paraffin embedding technique, was performed with a histopathological diagnosis of pleomorphic lacrimal gland adenoma. Histological sections were stained with Hematoxylin / Eosin. Immunostaining with Ki67 and MUC-1 was performed with the DAKO LSAB + kit. Results: The diagnosis of hypercellular pleomorphic adenoma with areas of the remaining lacrimal gland was made. Ki67 labeling was low $(\leq 15 \%)$. MUC-1 expression was intense, situated to the apical cell membrane of approximately $10 \%$ epitheliocytes from pseudoductal and cystic structures. Conclusions: Through histopathological evaluation, the correlation of Ki67 expression and the location and expression of MUC1, we verified that it is a non-recurrent pleomorphic adenoma without malignant transformation.
\end{abstract}

KEYWORDS: lacrimal gland, pleomorphic adenoma, histopathology, immunohistochemistry

\section{Resumen}

Introducción: En la práctica oftalmológica diaria los tumores de la glándula lagrimal son poco comunes. Representan el 5 a 7,5\% de todos los tumores intraorbitarios. El tumor epitelial más común de esta glándula es el adenoma pleomórfico, con un porcentaje de 25 al 50\% de sus lesiones tumorales Objetivo: En esta comunicación presentamos un caso de adenoma pleomórfico de glándula lagrimal y estudiamos la expresión de Ki67 y la localización y expresión de MUC-1 y su correlación con el pronóstico tumoral. Realizamos además un estudio descriptivo retrospectivo de la literatura sobre el tema publicada entre los años 1951 y 2020 , usando la base de datos MEDLINE. Material y métodos Se realizó el examen de la pieza quirúrgica, procesada según la técnica de inclusión en parafina, con diagnóstico histopatológico de adenoma pleomórfico de glándula lagrimal. Los cortes histológicos se colorearon con Hematoxilina/Eosina. Se realizó inmunomarcación con Ki67 y MUC-1, con el kit LSAB+ de DAKO. Resultados: Se realizó el diagnóstico de adenoma pleomórfico de tipo hipercelular con áreas de glándula lagrimal remanente. La marcación con Ki67 fue baja $(\leq 15 \%)$. La expresión con MUC-1 era intensa, localizada en la membrana celular apical de aproximadamente $10 \%$ de los epiteliocitos de las formaciones seudoductales y quísticas. Conclusiones: Por medio de la evaluación histopatológica, la correlación de la expresión de Ki67 y la localización y expresión de MUC1, comprobamos que se trata de un adenoma pleomórfico no recurrente y sin transformación maligna.

PALABRAS CLAVE: glándula lagrimal, adenoma pleomórfico, histopatología, inmunohistoquímica

Received 20 April 2020; Received in revised form 20 July 2020; Accepted 10 October 2020 


\section{Introducción}

La glándula lagrimal humana es una glándula exocrina tubuloalveolar que se sitúa en la región súpero externa de la órbita. Anatómicamente consiste de la glándula lagrimal principal y la glándula lagrimal accesoria. La parte principal está formada por varios lóbulos separados por tejido conectivo laxo. En los lóbulos se localizan los acinos serosos y los conductos intralobulares mientras que los conductos interlobulares se encuentran en el tejido conectivo. Estos conductos van a formar 12 conductos excretores que se abren en el fondo de saco conjuntival ${ }^{1,2}$.

Las lágrimas mantienen la salud de la superficie ocular y la visión normal; su secreción se produce por un mecanismo semejante al de la secreción de la saliva primaria ${ }^{3}$.

En la práctica oftalmológica diaria los tumores de la glándula lagrimal son poco comunes de observar. Representan el 5 a 7,5\% de todos los tumores intraorbitarios. El tumor epitelial más común de esta glándula es el adenoma pleomórfico, con un porcentaje de 25 al $50 \%$ de sus lesiones tumorales ${ }^{4,5}$.

El adenoma pleomórfico (ICD-O-Code 8940/0International Classification of Diseases for Oncology) ${ }^{6}$ es un tumor de estructura histológica compleja, con células ductales y mioepiteliocitos organizados en diferentes patrones morfológicos constituyendo conductos, nidos sólidos y láminas que coexisten con áreas estromales hialinizadas, condroides y mixoides. Si bien se lo clasifica como benigno, el adenoma pleomórfico puede causar problemas en su manejo clínico debido a su tendencia a recurrir y a su posible transformación maligna. Dicha recurrencia se relaciona con el desarrollo de un patrón multinodular, el predominio de estroma mixoide y la infiltración capsular ${ }^{7}$.

Sobre las bases de su apariencia histológica se lo clasifica en dos tipos: hipercelular y mixoide. El adenoma pleomórfico tipo celular o hipercelular es una variante donde predomina el componente epitelial en tanto que el adenoma pleomórfico tipo mixoide está formado principalmente por elementos mixoides o mixocondroides ${ }^{8}$.

Por otro lado, Ki67 es un marcador de proliferación celular que tiene utilidad como factor pronóstico de las neoplasias ${ }^{9}$. En cuanto a MUC-1, es una mucina humana asociada a membrana que en las neoplasias malignas se sobre-expresa y pierde su localización exclusivamente apical ${ }^{10}$.

En esta comunicación presentamos un caso de adenoma pleomórfico de glándula lagrimal y estudiamos la expresión de Ki67 y la localización y expresión de MUC-1 y su correlación con el pronóstico tumoral. Realizamos además un estudio descriptivo retrospectivo de la literatura sobre el tema publicada entre los años 1951 y 2020, usando la base de datos MEDLINE. Para la búsqueda se emplearon las siguientes palabras clave: pleomorphic adenoma, mixed tumor y lacrimal gland.

\section{Caso clínico}

Paciente mujer de 55 años de edad que concurrió a la consulta oftalmológica por una tumoración orbitaria supero-externa izquierda de 6 meses de evolución, acompañada de disminución de la visión, proptosis y dolor a la palpación. La tomografía computada de la órbita mostró una masa homogénea hiperdensa bien definida $\mathrm{y}$ circunscripta, de $3 \mathrm{~cm}$ de diámetro. Se indicó la resección de la tumoración.

Se realizó el examen de la pieza quirúrgica, procesada según la técnica de inclusión en parafina, con diagnóstico histopatológico de adenoma pleomórfico de glándula lagrimal.

Los cortes histológicos seriados, de $4 \mu \mathrm{m}$ de espesor se colorearon con Hematoxilina/Eosina. Se realizó la inmunomarcación con Ki67 y MUC-1, con el kit LSAB+ de DAKO ${ }^{11}$.

Ki67 detecta un antígeno nuclear cuya expresión proporciona una medida directa de la fracción de crecimiento tisular. La evaluación de la inmunomarcación se realizó por el contaje celular en campos de mayor aumento sin áreas de necrosis y homogéneas en la población celular a estudiar. Sobre cada campo se contabilizó el número total de células y las células marcadas con Ki67 y se aplicó la fórmula células marcadas/células totales por 100 para obtener el índice de proliferación. Siguiendo el St. Gallen Consensus de 2009 se clasifican los tumores como de baja, intermedia y alta proliferación de acuerdo al índice de marcación de Ki67 de $\leq 15 \%, 16-30 \%$ y $>30 \%$, respectivamente ${ }^{9}$. MUC-1 es una glucoproteína que se expresa en la membrana celular de los epiteliocitos orales y ductales humanos. En las células normales la 
expresión ocurre sólo en la membrana apical. Para el análisis comparativo, los tumores que mostraron inmunomarcación positiva en $>10 \%$ de las células se consideraron positivos. Para la evaluación semicuantitativa se clasificó la marcación en predominantemente membranosa (apical o circunferencial) o predominantemente difusa, con inmunorreactividad citoplasmática ${ }^{12}$.

Como control de la inmunomarcación se emplearon cortes histológicos del tumor que presentaban glándula lagrimal remanente (Fig. $1 \mathrm{~A}$ y B).

En los cortes coloreados con $\mathrm{H} / \mathrm{E}$ se observó una lesión nodular revestida parcialmente por una cápsula de tejido conectivo fibroso, constituida principalmente por una densa población celular con núcleos vesiculosos y citoplasma acidófilo, que formaban estructuras seudoductales (Fig. 2 A). También se encontraron abundantes formaciones quísticas de diferente tamaño, ocupadas por una secreción acidófila (Fig. 2B) y acúmulos celulares sólidos. Se identificaron además escasas zonas de estroma mixomatoso con focos de hialinización y metaplasia condroide y un área con formación de perlas córneas (Fig. 2B y 3A y B). Se realizó el diagnóstico de adenoma pleomórfico de tipo hipercelular con áreas de glándula lagrimal remanente.

La marcación con Ki67 fue baja $(\leq 15 \%)$. La expresión con MUC-1 era intensa, localizada en la membrana celular apical de aproximadamente $10 \%$ de los epiteliocitos de las formaciones seudoductales y quísticas (Fig. 4 A y B).

En la Tabla 1 se presentan las publicaciones sobre adenoma pleomórfico de glándula lagrimal recuperadas de la Base de datos MEDLINE, consultada por medio del motor de búsqueda de libre acceso PubMed.

Tabla 1: Casos comunicados de adenoma pleomórfico de glándula lagrimal entre 1951 y 2020 (Fuente: PubMed ${ }^{13}$ )

\begin{tabular}{|l|l|l|}
\hline AUTORES & REVISTA & IDIOMA \\
\hline $\begin{array}{l}\text { Mc Kinney y } \\
\text { Butz }\end{array}$ & Am J Ophthalmol 34: 1519-1522, 1951 & Inglés \\
\hline $\begin{array}{l}\text { Markey } \\
\text { Zelaski }\end{array}$ & Bull Kresge Eye Inst 3: 67-71, 1952 & Inglés \\
\hline Werne & Brooklyn Hosp 12: 53-55, 1954 & Inglés \\
\hline Davies & $\begin{array}{l}\text { Trans Am Ophthalmol Soc 52: 467-496, } \\
\text { 1954- 1955 }\end{array}$ & Inglés \\
\hline Pal & Burma Med J 4: 21-23, 1956 & Inglés \\
\hline
\end{tabular}

\begin{tabular}{|c|c|c|}
\hline Graziani & Arcisp S Anna Ferrara 10:791-810, 1957 & Italiano \\
\hline Costner & $\begin{array}{l}\text { J Tnn State Med Assoc 51: 373-374, } \\
1958\end{array}$ & inglés \\
\hline Frera & $\begin{array}{l}\text { Ann Ottalmol Clin Ocul 84: 568-577, } \\
1958\end{array}$ & Italiano \\
\hline Vancea et al & $\begin{array}{l}\text { Arch Ophtalmol Rev Gen Ophtalmol 19: } \\
861-866,1959\end{array}$ & Francés \\
\hline $\begin{array}{l}\text { Agarwal y } \\
\text { Dhanda }\end{array}$ & $\begin{array}{l}\text { J All India Ophthalmol Soc 9: 97-99, } \\
1961\end{array}$ & Inglés \\
\hline Cheng & Chin Med J 83: 49-51, 1964 & Inglés \\
\hline Shiga y Morita & Nihon Ganka Kiyo 15: 28-30, 1964 & Japonés \\
\hline $\begin{array}{l}\text { Linsell } \quad y \\
\text { Clifford }\end{array}$ & East Afr Med J 41: 263-270, 1964 & Inglés \\
\hline $\begin{array}{l}\text { Vancea } \\
\text { Vrejoiu }\end{array}$ & $\begin{array}{l}\text { Arch Ophtalmol Rev Gen Ophtalmol 24: } \\
691-695,1964\end{array}$ & Francés \\
\hline Böck y Feyrter & Ophthalmologica 151: 331-348, 1966 & Alemán \\
\hline Böck y Feyrter & Doc Ophthalmol 24: 275-288, 1968 & Alemán \\
\hline $\begin{array}{l}\text { Bouchayer y } \\
\text { Oudot }\end{array}$ & $\begin{array}{l}\text { J Fr Otorhinolaryngol Audiophonol Chir } \\
\text { Maxillofac 19: 268-272, } 1970\end{array}$ & Francés \\
\hline Filipic & $\begin{array}{l}\text { Trans Aust Coll Ophthalmol 3: 64-71, } \\
1971\end{array}$ & Inglés \\
\hline Kapoor et al & Indian J Ophthalmol 25: 52-53, 1978 & Inglés \\
\hline Mueller y Borit & Ann Ophthalmol 11: 661-663, 1979 & Inglés \\
\hline Saini et al & Indian J Ophthalmol 33: 181-182, 1985 & Inglés \\
\hline Desouza et al & J Postgrad Med 33: 41-42, 1987 & Inglés \\
\hline Shields et al & Arch Ophthalmol 105: 560-561, 1987 & Inglés \\
\hline Shields y Shields & Arch Ophthalmol 105: 1403-1405, 1987 & Inglés \\
\hline Auran et al & Ophthalmology 95: 90-99, 1988 & Inglés \\
\hline Shields y Shields & $\begin{array}{l}\text { Trans Pa Acad Ophthalmol Otolaryngol } \\
\text { 42: 925-930, } 1990\end{array}$ & Inglés \\
\hline Riedel et al & Neurosurg Rev 13: 289-298, 1990 & Inglés \\
\hline Tosaka y Fujii & $\begin{array}{l}\text { Nippon Ganka Gakkai Zasshi 94: 740- } \\
745,1990\end{array}$ & Japonés \\
\hline Yamasaki et al & $\begin{array}{l}\text { Neurol Med Chir (Tokyo) 30: 1038- } \\
1042,1990\end{array}$ & Japonés \\
\hline Tosaka & Jpn J Ophthalmol 4: 367-376, 1991 & Inglés \\
\hline Kamei et al & No Shinkei Geka 20: 79-83, 1992 & Japonés \\
\hline D`Hermies et al & J French Ophtalmol 15: 220-223, 1992 & Francés \\
\hline Rose y Wright & British J Ophthalmol 76: 395-400, 1992 & Inglés \\
\hline Singh et al & Indian J Ophthalmol 40: 118-121, 1992 & Inglés \\
\hline Miller & British J Ophthalmol 77: 464-465, 1993 & Inglés \\
\hline Tsunoda et al & $\begin{array}{l}\text { Neurol Med Chir (Tokyo) 34: 814-816, } \\
1994\end{array}$ & Inglés \\
\hline Ostrowsky et al & Ophthalmology 101: 925-930, 1994 & Inglés \\
\hline Christie et al & Am J Ophthalmol 119: 97-99, 1995 & Inglés \\
\hline Pârgă & Oftalmologia 39: 137-140, 1995 & Rumano \\
\hline Zheng et al & $\begin{array}{l}\text { Zhonghua Yan Ke Za Zhi 31: 212-214, } \\
1995\end{array}$ & Chino \\
\hline Tong et al & $\begin{array}{l}\text { Ophthalmic Plast Reconstr Surg 11: 136- } \\
138,1995\end{array}$ & Inglés \\
\hline Zheng et al & $\begin{array}{l}\text { Zhonghua Yan Ke Za Zhi 32: 133-135, } \\
1996\end{array}$ & Chino \\
\hline
\end{tabular}




\begin{tabular}{|c|c|c|}
\hline Zheng et al & Yan ke Xue Bao 12: 54-57, 1996 & Chino \\
\hline Faktorovich et al & Am J Ophthalmol 122: 446-447, 1996 & Inglés \\
\hline Pe'er et al & Ophthalmology 103: 1601-1605, 1996 & Inglés \\
\hline $\begin{array}{l}\text { Vangveeravong } \\
\text { et al }\end{array}$ & Ophthalmology 103: 1606-1612, 1996 & Inglés \\
\hline Tang et al & $\begin{array}{l}\text { Zhonghua Yan Ke Za Zhi 33: 354-356, } \\
1997\end{array}$ & Chino \\
\hline Uehara et al & $\begin{array}{l}\text { Nippon Ganka Gakkai Zasshi 101: 355- } \\
358,1997\end{array}$ & Japonés \\
\hline Mercado et al & Arch Ophthamol 116: 962-963, 1998 & Inglés \\
\hline Motegi et al & Jpn J Ophthalmol 42: 249-255, 1998 & Inglés \\
\hline $\begin{array}{l}\text { Wharton y } \\
\text { O'Donell }\end{array}$ & $\begin{array}{l}\text { Aust N Z J Ophthalmol 27: 145-148, } \\
1999\end{array}$ & Inglés \\
\hline Yamada et al & Ophthalmologica 213: 269-272, 1999 & Inglés \\
\hline Paulino et al & Ann Diagn Pathol 3: 199-204, 1999 & Inglés \\
\hline Gierek et al & Otolaryngol Pol 53: 323-325, 1999 & Polaco \\
\hline Guerra et al & $\begin{array}{l}\text { J Oral Maxillofac Surg 58: 569-572, } \\
2000\end{array}$ & Inglés \\
\hline Hsu & Ophthalmologica $215: 235-237,2001$ & Inglés \\
\hline Lakhey et al & $\begin{array}{l}\text { Indian J Pathol Microbiol 44: 333-335, } \\
2001\end{array}$ & Inglés \\
\hline $\begin{array}{l}\text { Chandrasekhar } \\
\text { et al }\end{array}$ & $\begin{array}{l}\text { Br J Oral Maxillofac Surg 39: 390-393, } \\
2001\end{array}$ & Inglés \\
\hline Cates et al & Br J Ophthalmol 86: 249-250, 2002 & Inglés \\
\hline Becelli et al & J Craniofac Surg 13: 49-52, 2002 & Inglés \\
\hline Baredes et al & Laryngoscope 113: 940-942, 2003 & Inglés \\
\hline Sadick et al & $\begin{array}{l}\text { ORL J Otorhinolaryngol Relat Spec } 65 \text { : } \\
295-299,2003\end{array}$ & Inglés \\
\hline Fenton et al & Eye (London) 18: 77-79, 2004 & Inglés \\
\hline $\begin{array}{l}\text { Kuroishikawa et } \\
\text { al }\end{array}$ & Jpn J Ophthalmol 48: 181-182, 2004 & Inglés \\
\hline Stefko et al & Arch Ophthalmol 122: 778-780, 2004 & Inglés \\
\hline Zhu et al & $\begin{array}{l}\text { Zhonghua Yan Ke Za Zhi 40: 220-224, } \\
2004\end{array}$ & Chino \\
\hline Stupp et al & Arch Ophthalmol 122: 1538-1540, 2004 & Inglés \\
\hline Sen et al & Clin Exp Ophthalmol 32: 523-525, 2004 & Inglés \\
\hline Halhal et al & J Fr Ophtalmol 27: 1200-1203, 2004 & Francés \\
\hline Marshall et al & $\begin{array}{l}\text { Otolaryngol Head Neck Surg 132: } 141 \text { - } \\
143,2005\end{array}$ & Inglés \\
\hline Yilmaz et al & $\begin{array}{l}\text { Turkish Neurosurgery 15: 140-143, } \\
2005\end{array}$ & Inglés \\
\hline Ostrosky et al & $\begin{array}{l}\text { Med Oral Patol Oral Cir Bucal 10: 88- } \\
89 ; 86-88,2005\end{array}$ & $\begin{array}{l}\text { Inglés, } \\
\text { Español }\end{array}$ \\
\hline Fichter et al & Ophthalmologe 102: 399-423, 2005 & Alemán \\
\hline Hajda et al & Magy Onkol 49: 65-70, 2005 & Húngaro \\
\hline Miyazaki et al & $\begin{array}{l}\text { Neurol Med Chr (Tokyo) 45: 407-410, } \\
2005\end{array}$ & Inglés \\
\hline
\end{tabular}

\begin{tabular}{|c|c|c|}
\hline $\begin{array}{ll}\text { Lacrymal } & \text { Gland } \\
\text { Tumor } & \text { Study } \\
\text { Group } & \\
\end{array}$ & Jpn J Ophthalmol 49: 343-348, 2005 & Inglés \\
\hline Galatoire et al & J Fr Ophtalmol 28: 896-901, 2005 & Frances \\
\hline Bi y Chen & $\begin{array}{l}\text { Zhonghua Yan Ke Za Zhi 41: 877-881, } \\
2005\end{array}$ & Chino \\
\hline Chen et al & Clin Exp Ophthalmol 33: 639-641, 2005 & Inglés \\
\hline Mensink et al & Clin Exp Ophthalmol 33: 669-671, 2005 & Inglés \\
\hline Perez et al & $\begin{array}{l}\text { Otolaryngol Head Neck Surg 134: 321- } \\
325,2006\end{array}$ & Inglés \\
\hline Obata & Cornea 25: s82-s89, 2006 & Inglés \\
\hline $\begin{array}{l}\text { Hadjistilianou et } \\
\text { al }\end{array}$ & Orbit 25: 51-53, 2006 & Inglés \\
\hline Alyahya et al & Ophthalmology 113: 879-882, 2006 & Inglés \\
\hline Chang et al & $\begin{array}{l}\text { Ann Ophthalmol (Skokie) 38: 141-144, } \\
2006\end{array}$ & Inglés \\
\hline Porter et al & $\begin{array}{l}\text { Otolaryngol Head Neck Surg 136: 328- } \\
329,2007\end{array}$ & Inglés \\
\hline Gibson et al & $\begin{array}{l}\text { Ophthalmic Plast Reconstr Surg 23: } 234 \\
-235,2007\end{array}$ & Inglés \\
\hline Ramlee et al & Orbit 26: $137-139,2007$ & Inglés \\
\hline Reza Vagefi et al & $\begin{array}{l}\text { Ophthalmic Plast Reconstruct Surg 23: } \\
\text { 272-274, } 2007\end{array}$ & Inglés \\
\hline Takahira et al & $\begin{array}{l}\text { Ophthalmic Plast Reconstr Surg 23: } \\
407-409,2007\end{array}$ & Inglés \\
\hline Currie et al & Arch Ophthalmol 125: 1643-1646, 2007 & Inglés \\
\hline Sakuma et al & Jpn J Ophthalmol 52: 67-68, 2008 & Inglés \\
\hline $\begin{array}{l}\text { Orzechowska- } \\
\text { Wylegala et al }\end{array}$ & Klin Oczna 110: 301-303, 2008 & Inglés \\
\hline Liao et al & Exp Eye Res 88: 4-11, 2009 & Inglés \\
\hline Lai et al & Eye (London) 23: 2-6, 2009 & Inglés \\
\hline Weis et al & Arch Ophthalmol 127: 1016-1028, 2009 & Inglés \\
\hline Rose & Eye (London) 23: 1625-1628, 2009 & Inglés \\
\hline Ishida et al & Rinsho Byori 57: 746-751, 2009 & Inglés \\
\hline Kopp et al & Eye (London) 24: 386, 2010 & Inglés \\
\hline Zeng et al & $\begin{array}{l}\text { Graefes Arch Clin Exp Ophthalmol 248: } \\
1345-1349,2010\end{array}$ & Inglés \\
\hline Patyal et al & Indian J Ophthalmol 58: 245-247, 2010 & Inglés \\
\hline $\begin{array}{l}\text { Prabhakaran et } \\
\text { all }\end{array}$ & Br J Ophthalmol 94: 1509-1512, 2010 & Inglés \\
\hline Civit et al & Neurochirurgie 56: 152-157, 2010 & Francés \\
\hline Chowdhury & Indian J Ophthalmol 59: 167, 2011 & Inglés \\
\hline Mc Nab y Satchi & Ophtalmology 118: 2088-2092, 2011 & Inglés \\
\hline Kini et al & Oral Maxillofac Surg 16: 123-126, 2012 & Inglés \\
\hline $\begin{array}{l}\text { Santaliz-Ruiz et } \\
\text { al }\end{array}$ & $\begin{array}{llrl}\text { Case Rep Med 2012; } & \text { doi: } \\
10.1155 / 2012 / 148103 & & \\
\end{array}$ & Inglés \\
\hline $\begin{array}{l}\text { Pointdujour y } \\
\text { Shinder }\end{array}$ & Ophthalmology 119: 1293-1294, 2012 & Inglés \\
\hline Rinna et al & $\begin{array}{l}\text { Eur Rev Med Pharmacol Sci 16: 90-94, } \\
2012\end{array}$ & Inglés \\
\hline Alsuhaibani & Saudi J Ophthalmol 26: 453-455, 2012 & Inglés \\
\hline
\end{tabular}




\begin{tabular}{|c|c|c|}
\hline $\begin{array}{l}\text { Shalinee Rao et } \\
\text { al }\end{array}$ & Brief Comm 62: 743-746, 2013 & Inglés \\
\hline Gupta et al & Diagn Cytopathol 41: 565-566, 2013 & Inglés \\
\hline $\begin{array}{l}\text { Von Holstein et } \\
\text { al }\end{array}$ & Acta Ophthalmol 91: 195-206, 2013 & Inglés \\
\hline Tailor et al & Radiographics 33: 1739-1758, 2013 & Inglés \\
\hline Obi et al & $\begin{array}{l}\text { Ophthalmic Plast Reconstr Surg 29: e4- } \\
\text { e7, } 2013\end{array}$ & Inglés \\
\hline Labh Y Shrestha & $\begin{array}{l}\text { JNMA J Nepal Med Assoc 52: 505-507, } \\
2013\end{array}$ & Inglés \\
\hline Binatli et al & $\begin{array}{l}\text { J SurgmCase } \quad \text { Rep } \quad 2013 ; \\
\text { doi:10.1093/jscr/rjt089 }\end{array}$ & Inglés \\
\hline Huang et al & Am J Med Sci 346: 162-163, 2013 & Inglés \\
\hline $\begin{array}{ll}\text { Gupta } & \text { y } \\
\text { Khandelwal } & \end{array}$ & $\begin{array}{l}\text { BMJ Case Report 2013; doi } \\
: 10.1136 / \text { bcr-2013-009138 }\end{array}$ & Inglés \\
\hline Vijayakumar & J Clin Diagn Res 7: 712-714, 2013 & Inglés \\
\hline Iyeyasu et al & Rev Bras Oftalmol 72: 338-340, 2013 & Inglés \\
\hline Mendoza et al & Am J Ophthalmol 156: 1147-1158, 2013 & Inglés \\
\hline Tang et al & Chin J Cancer Res 25: 423-429, 2013 & Inglés \\
\hline Chawla et al & $\begin{array}{l}\text { Ophthal Plast Reconstr Surg 29: 440- } \\
445,2013\end{array}$ & Inglés \\
\hline Von Holstein & Acta Ophthalmol 6: 1-28, 2013 & Inglés \\
\hline $\begin{array}{l}\text { Von Holstein et } \\
\text { al }\end{array}$ & Ophthalmology 121: 1125-1133, 2014 & Inglés \\
\hline Bae y Kim & $\begin{array}{l}\text { J Korean Ophthalmol Soc 55: 422-425, } \\
2014\end{array}$ & Inglés \\
\hline $\begin{array}{l}\text { Elouarradi y } \\
\text { Daoudi }\end{array}$ & Pan Afr Med 17: 114, 2014 & Inglés \\
\hline Pokharel et al & J Nepal Med Assoc 52: 949-951, 2014 & Inglés \\
\hline Daniel et al & Indian J Ophthalmol 62: 743-746, 2014 & Inglés \\
\hline Kurniawan et al & Clin Exp Ophthalmol 43: 591-592, 2015 & Inglés \\
\hline Yan et al & J Craniofac Surg 26: e187- e188, 2015 & Inglés \\
\hline Korchak et al & Orbit 34: 112-114, 2015 & Inglés \\
\hline Casado et al & $\begin{array}{l}\text { Arch Soc Esp Oftalmol 90: 432-434, } \\
2015\end{array}$ & Inglés \\
\hline Lee et al & Indian J Ophthalmol 63: 282-284, 2015 & Inglés \\
\hline Sung et al & $\begin{array}{l}\text { J Korean Neurosurg Soc 57: 473-477, } \\
2015\end{array}$ & Inglés \\
\hline Gonçalves et al & $\begin{array}{l}\text { Ophthalmic Plast Reconstr Surg 32: e42- } \\
\text { e44, } 2016\end{array}$ & Inglés \\
\hline Adekunle et al & $\begin{array}{l}\text { Ophthalmic Plast Reconstr Surg 32: e18- } \\
\text { e21, } 2016\end{array}$ & Inglés \\
\hline Xiao & $\begin{array}{l}\text { Zhonghua Yan Ke Za Zhi 52: 241-243, } \\
2016\end{array}$ & Chino \\
\hline Watanabe et al & Jpn J Ophthalmol 60: 286-293, 2016 & Inglés \\
\hline Andreasen et al & Acta Ophthalmol 94: 798-804, 2016 & Inglés \\
\hline Misra et al & Orbit 35: 295-297, 2016 & Inglés \\
\hline Rothwell et al & $\begin{array}{l}\text { J Ophthalmic Vis Res. 11:439-441, } \\
\text { 2016; doi: } 10.4103 / 2008-322 X .194144\end{array}$ & Inglés \\
\hline Jakobiec et al & Surv Ophthalmol 62: 219-226, 2017 & Inglés \\
\hline Wang et al & Ophthalmology 124: 952, 2017 & Inglés \\
\hline
\end{tabular}

\begin{tabular}{|c|c|c|}
\hline Yao y Zhang & $\begin{array}{ll}\text { Biotechnol Lett } & \text { 2017; } \\
\text { doi:10.1007/s10529-017-2326-1 } & \end{array}$ & Inglés \\
\hline Pushker et al & Can J Ophthalmol 52: e7-e9, 2017 & Inglés \\
\hline Skippen et al & Int Ophthalmol 38: 381-384, 2018 & Inglés \\
\hline Alam et al & Orbit 37: 125-127, 2018 & Inglés \\
\hline Pakdel et al & $\begin{array}{l}\text { Ophthalmic Plast Reconstr Surg 34: e41- } \\
\text { e43, } 2018\end{array}$ & Inglés \\
\hline $\begin{array}{l}\text { Larbcharoensub } \\
\text { et al }\end{array}$ & Mol Clin Oncol 8: 209-213, 2018 & Inglés \\
\hline Andreasen et al & Acta Ophthalmol 96: e768-e771, 2018 & Inglés \\
\hline Harrison & Saudi J Ophthalmol 32: 13-16, 2018 & Inglés \\
\hline Mulay et al & Acta Ophthalmol 96: e772-e775, 2018 & Inglés \\
\hline Wajda et al & Int Ophthalmol 39: 1617-1619, 2018 & Inglés \\
\hline $\begin{array}{l}\text { Bhuyan y } \\
\text { Varrosang Varte }\end{array}$ & Indian J Appl Res 9: 25-26, 2019 & Inglés \\
\hline Young et al & Br J Ophthalmol 103: 264-268, 2019 & Inglés \\
\hline Clarós et al & Acta Otolaryngol 139: 100-104, 2019 & Inglés \\
\hline Porto et al & Cir Parag 44: 27-28, 2019 & Inglés \\
\hline Ahn et al & $\begin{array}{l}\text { Graefes Arch Clin Exp Ophthalmol 257: } \\
1527-1533,2019\end{array}$ & Inglés \\
\hline Zhang et al & Int J Ophthalmol 12: 1238-1242, 2019 & Inglés \\
\hline Bryant et al & $\begin{array}{l}\text { Plast Reconstr Surg Glob Open 7: e2435, } \\
2019 \text {, } \\
\text { 10.1097/GOX.0000000000002435 }\end{array}$ & Inglés \\
\hline Garakani et al & $\begin{array}{l}\text { Ophthalmic Plast Reconstr Surg 36: e1- } \\
\text { e3, } 2020\end{array}$ & Inglés \\
\hline Ayala et al & Rev Esp Patol 53: 55-60, 2020 & Inglés \\
\hline $\begin{array}{l}\text { Schittkowski y } \\
\text { Storch }\end{array}$ & $\begin{array}{l}\text { Klin Monbl Augenheilkd 2020; doi } \\
: 10.1055 / \mathrm{a}-1068-7699\end{array}$ & Inglés \\
\hline
\end{tabular}

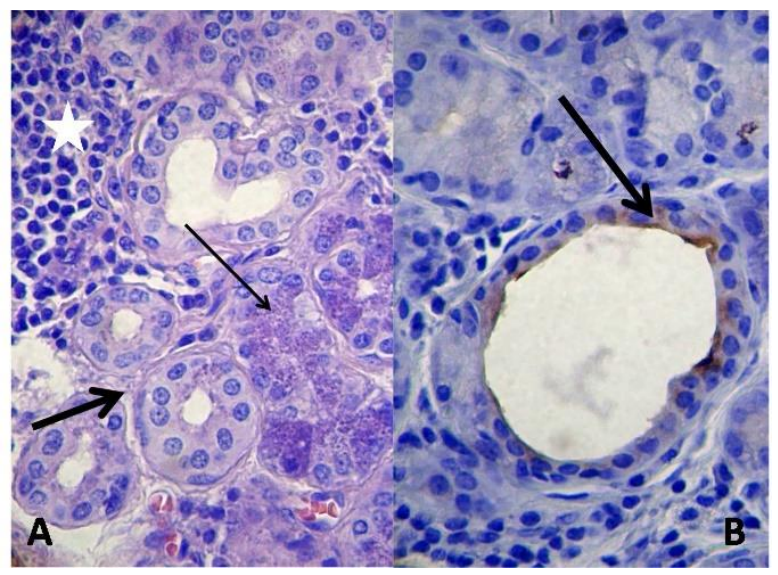

Figura 1. Glándula Lagrimal. A) Se señalan los acinos serosos (flecha fina) y los conductos interlobulillares (flecha gruesa). Foco de infiltrado inflamatorio (estrella). H/E. Objetivo 40 x. B) La flecha gruesa señala la marcación apical positiva de un conducto interlobulillar. Inmunomarcación MUC-1. Objetivo 40 x 


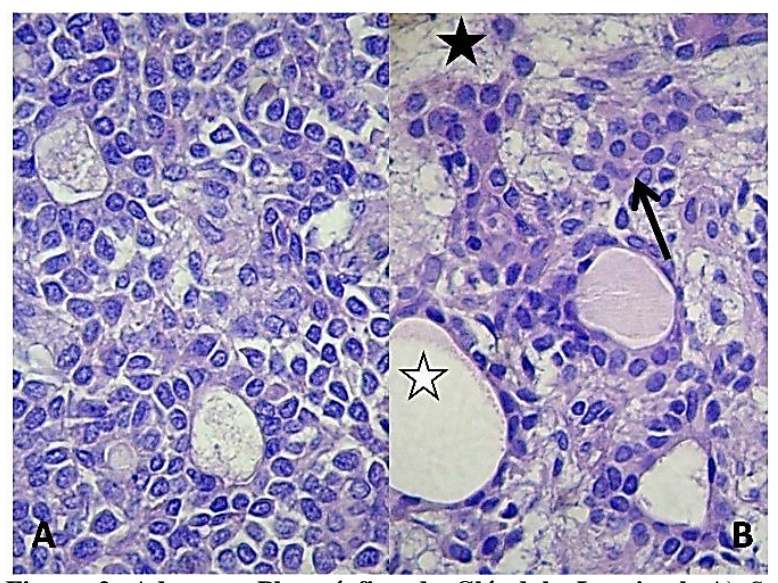

Figura 2. Adenoma Pleomórfico de Glándula Lagrimal. A) Se observa la lesión formada principalmente por una densa población celular. H/E. Objetivo 40 x. B) Formaciones quísticas ocupadas por secreción acidófila (estrella blanca). Acúmulos celulares sólidos (flecha). Zona de estroma mixoide (estrella negra). H/E. Objetivo 40 $\mathrm{x}$

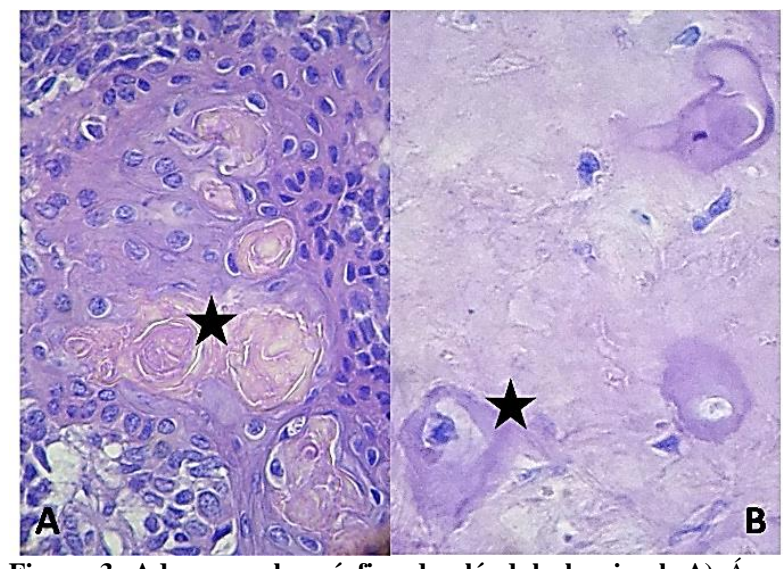

Figura 3. Adenoma pleomórfico de glándula lagrimal. A) Área tumoral con formación de perlas córneas (estrella). H/E. Objetivo 40 x. B) Foco de metaplasia condroide (estrella). H/E. Objetivo $40 \mathrm{x}$

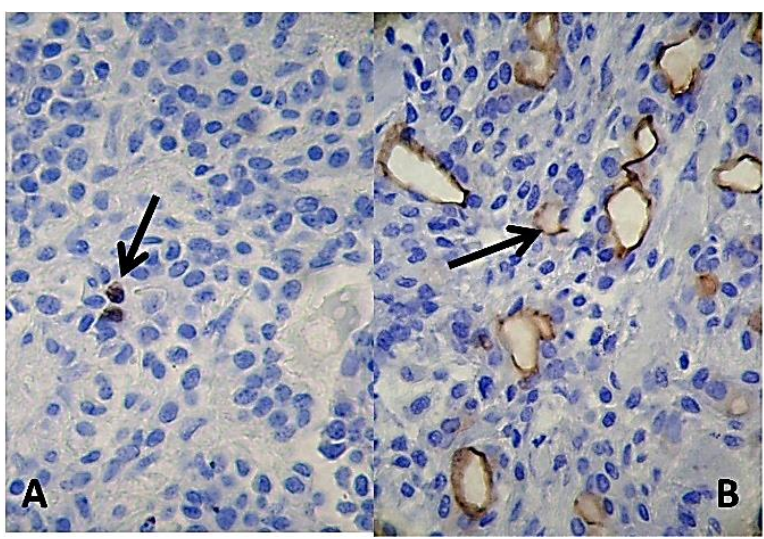

Figura 4. Adenoma Pleomórfico de Glándula Lagrimal. A) Se señala la baja marcación con Ki67 (flecha). Inmunomarcación Ki67. Objetivo 40 x. B) Expresión intensa con MUC-1 localizada en la membrana apical de los epiteliocitos en formaciones seudoductales y quísticas (flecha). Inmunomarcación MUC-1. Objetivo 40 x

\section{Aspectos éticos}

Este trabajo es parte del proyecto "La matriz extracelular y el componente mioepitelial de los tumores epiteliales de glándulas salivales humanas: estudio estructural, histoquímico, e inmunohistoquímico (Subsidio Consolidar SECYT Res 411/2018). Su aprobación ética fue obtenida del Comité de Ética del Hospital Nacional de Clínicas. Facultad de Ciencias Médicas. Universidad Nacional de Córdoba. Argentina (Registro 188/14).

\section{Discusión}

En la órbita de los individuos adultos se localiza una gran variedad de tumores benignos y malignos. Entre ellos, las lesiones de las glándulas lagrimales, que son comparativamente poco comunes en la práctica oftalmológica clínica; corresponden a un 5 a $14 \%$ de las patologías del espacio orbitario, representando los tumores epiteliales un $30 \%$ del total ${ }^{14-16}$.

El adenoma pleomórfico es el tumor de glándulas salivales más común, tanto en niños como en adultos, y puede presentar localizaciones extrasalivales. A nivel orbitario comprende el $12 \%$ de las lesiones epiteliales benignas de la glándula lagrimal ${ }^{6,15}$.

El caso relatado en el presente trabajo corresponde a una paciente de 55 años, edad que coincide con la literatura ya que se considera que ocurre más comúnmente entre la segunda y quinta década de la vida ${ }^{17}$.

Sobre las bases de su apariencia histológica y la proporción relativa entre el componente epitelial y el estroma se determinó que correspondía a un adenoma pleomórfico predominantemente celular con escasos focos de componentes mixoide, condroide y hialino ${ }^{8}$.

Su diagnóstico diferencial abarca varias patologías como el linfoma, el carcinoma adenoide quístico, la dacrioadenitis crónica, la sarcoidosis, el hemangioma cavernoso, el síndrome de Sjögren y el schwannoma intralagrimal, como relatan Iyeyasu et $\mathrm{al}^{18}$.

Si bien el adenoma pleomórfico se clasifica como un tumor benigno, tiene tendencia a recurrir después de su primer tratamiento quirúrgico, con riesgo de transformación maligna ${ }^{19,20}$. 
Esta recurrencia se produce principalmente por diferencias en su abordaje quirúrgico, la ruptura intra-quirúrgica, sus características histopatológicas o el desarrollo de seudopodos como mencionan Borges Soares et al y Henriksson et $\mathrm{al}^{20,21}$.

Por otro lado, la mucina humana MUC-1 asociada a membrana fue descubierta hace unos 40 años como un componente de los glóbulos de grasa de la leche y ha modificado su nombre muchas veces. Antígeno epitelial de membrana (EMA), PAS-O, antígeno DF3, antígeno $\mathrm{H} 23$, glucoproteína no penetrante (NPGP), PEM, antígeno NCRC11 son algunas de las denominaciones de esta molécula ${ }^{10}$, 22 .

En la literatura se encuentran numerosos trabajos de la expresión de esta mucina en distintos tipos de tumores $^{23-25}$. Por el contrario, no se encontraron investigaciones a nivel de los tumores de la glándula lagrimal ni su asociación con Ki67.

En nuestra investigación la inmunomarcación de MUC-1 en la glándula lagrimal normal se expresó en la superficie apical de los conductos interlobulillares y sólo ocasionalmente en la membrana apical de las células de los acinos serosos como se puede observar en la Figura 1A, a diferencia de los resultados obtenidos por Paulsen et $\mathrm{al}^{26}$, quienes describen la positividad de la marcación a nivel de la superficie apical de las células acinares serosas.

En las células tumorales la reacción fue positiva solamente en la membrana apical de un $10 \%$ de los epiteliocitos localizados en las estructuras quísticas y seudoductales. Hamada et $\mathrm{al}^{27}$ estudiaron la expresión de MUC-1 como marcador de recurrencia en el adenoma pleomórfico de glándulas salivales. Encontraron que estaba sobre expresada en varios casos de adenoma pleomórfico y consideraron que la elevada expresión de este antígeno actuaba como un factor independiente para predecir la recurrencia tumoral.

También Borges Soares et $\mathrm{al}^{20}$ estudiaron la expresión de MUC-1 en diferentes fases de la secuencia adenoma-carcinoma; analizaron 21 casos de adenoma pleomórfico, 18 casos de adenoma pleomórfico recurrente, 3 casos de adenoma pleomórfico recurrente con transformación focal y 11 casos de carcinoma ex adenoma pleomórfico. Estos autores encontraron importantes diferencias en todos los grupos investigados y concluyeron que la sobre expresión de esta molécula se asocia con la recurrencia tumoral y su transformación maligna.

Gao et $\mathrm{a}^{28}$ también consideran que la anormal distribución y expresión de MUC-1 contribuye al carácter invasor del adenoma pleomórfico y puede emplearse como una señal predictiva de recurrencia tumoral.

Este estudio es de significación para el pronóstico y tratamiento de este tumor salival, y resultan de interés su extrapolación a otras localizaciones como el relatado por nosotros en glándula lagrimal ya que no se encontraron en la literatura investigaciones comparativas con MUC-1.

Además, en relación a la capacidad proliferativa de la lesión de glándula lagrimal estudiada con Ki67, la misma mostró una baja positividad.

Zhang et $\mathrm{al}^{29}$ compararon la expresión de Ki67 en el adenoma pleomórfico y el carcinoma ex adenoma pleomórfico de glándula lagrimal demostrando la baja positividad del adenoma pleomórfico que contrastaba de manera manifiesta con la alta expresión observada en el carcinoma ex adenoma pleomórfico, marcador de valor para su diagnóstico diferencial.

Como mencionamos en la Introducción MUC-1 asociada a la membrana se sobre expresa en las neoplasias malignas perdiendo su localización exclusivamente apical. Ocupa toda la superficie de la membrana, el citoplasma de las células tumorales e incluso el núcleo ${ }^{10,30}$.

En nuestro estudio comprobamos por medio de la evaluación histopatológica, la correlación de la expresión de Ki67 y la localización y expresión de MUC-1, que se trata de un adenoma pleomórfico no recurrente y sin transformación maligna. Se aconseja el seguimiento y control de la paciente para detectar posibles recurrencias o su derivación en un carcinoma ex adenoma pleomórfico.

Todos los autores declaran que no existen conflictos potenciales de interés con respecto a la autoría y / o publicación de este artículo. All authors declare no potential conflicts of interest with respect to the authorship and/or publication of this article.

\section{Agradecimientos}

El presente trabajo fue realizado bajo el apoyo de la Secretaría de Ciencia y Tecnología de la Universidad Nacional de Córdoba, Argentina (proyecto SECYT-UNC Resolución 411/18). 


\section{Referencias}

1. Obata H. Anatomy and histopathology of the human lacrimal gland. Cornea 2006; 25: S82-S89.

2. Yao Y, Zhang Y. The lacrimal gland: development, wound repair and regeneration. Biotechnol Lett 2017; DOI 10.1007/s10529-017-2326-1.

3. Welsch U, Deller T. Sobotta. Histología. $3^{\circ}$ ed. México: Ed Médica Panamericana, 2013: 513-514.

4. Ayala PE, Dermith AM, Antúnez HS, Murillo TP. Adenoma pleomorfo de glándula lagrimal: reporte de un caso pediátrico. Rev Esp Patol 2020; 53: 55-60

5. Porto M, Pane M, García M, Mussi D, Aquino Caballero O. Adenoma pleomorfo de glándula lagrimal: a propósito de un caso. Cir Parag 2019; 44: 27-28.

6. Organización Mundial de la Salud. WHO/IARC. Classification of Head and Neck Tumours. 4th ed. WHO. Lyon: Edited by El-Naggar AK, Chan JKC, Grandis JR, Takata T, Slootweg PJ, 2017.

7. Samar ME, Avila RE. Tumores Epiteliales de Glándulas Salivales. Saarbrücken. Alemania: Editorial Académica Española, 2013.

8. Ellis GL, Auclair PL. Tumors of salivary glands. Washington, DC: American Registry of Pathology Armed Forces Institute of Pathology, 2008.

9. Li LT, Jiang G, Chen Q, Zheng, JN. Ki67 is a promising molecular target in the diagnosis of cancer. Mol. Med. Reports 2015; 11: 1566-1572.

10. Syrkina, MS, Vassetzky YS, Rubtsov MA. MUC-1 story: great expectations, disappointments and the renaissance. Current Med. Chem 2017; 24: 1-10.

11. Samar ME, Avila RE, Esteban Ruiz F. Técnicas histológicas. Fundamentos y aplicaciones. Córdoba: Editorial SeisC, 2004.

12. Langner C, Ratschek M, Rehak P, Schips L, Zigeuner R. Expression of MUC-1 (EMA) and E-cadherin in renal cell carcinoma: a systematic immunohistochemical analysis of 188 cases. Modern Pathol 2004; 7: 180-188.

13. PUBMED. https://www.ncbi.nlm.nih.gov/pubmed. Consulta: 18/02/2020. (Última consulta: 10 de abril de 2020).

14. Tailor TD, Gupta D, Dalley RW, Keene D, Anzai Y. Orbital neoplasms in adults: clinical, radiologic and pathologic review. Radiographics 2013; 33: 1739-1758.

15. Binatli O, Yaman O, Ozdemir N, Gokcol Erdogan I. Pleomorphic adenoma of lacrimal gland. J Surg Case Rep 2013; doi:10.1093/jscr/rjt089

16. Schittkowski MP y Storch MW. Diseases of the lacrimal gland. Klin Monbl Augenheilkd 2020; doi :10.1055/a1068-76992020

17. Bhuyan J y Varrosang Varte D. Pleomorphic adenoma of lacrimal gland with intraconal extension- A rare case report. Indian J Appl Res 2019; 9: 25-26.
18. Iyeyasu JN, Reis F, Altemani AM, Monteiro de Carvalho K.An unusual presentation of lacrimal gland pleomorphic adenoma. Rev Bras Oftalmol 2013; 72: 338340 .

19. Currie ZI y Rose GE. Long-term risk of recurrence after intact excision of pleomorphic adenomas of the lacrimal gland. Arch Ophthalmol 2007; 125: 1643-1646.

20. Borges Soares A, Dias Demasi AP, Altemani A, Calvacanti de Araujo V. Increased mucin 1 expression in recurrence and malignant transformation of salivary gland pleomorphic adenoma. Histopathology 2011; 58: 377-382.

21. Henriksson G, Westrin KM, Carlsoo B, Silfversward C. Recurrent primary pleomorphic adenomas of salivary gland origin: intrasurgical rupture, histopathologic features and pseudopodia. Cancer 1998; 82: 617-620.

22. Ponce-Bravo S, Ledesma-Montes C, Morales- Sánchez I. MUC-1 mucin in normal human salivary glands detected by HMFG-1 and HMFG-2 monoclonal antibodies. APMIS 2008; 116: 93-98.

23. Lau SK, Weiss LM, Chu PG. Differential expression of MUC1, MUC2 and MUC5AC in carcinomas of various sites. An immunohistochemical study. Am J Clin Pathol 2004; 122: 61-69.

24. Behera SK, Praharaj AB, Dehury B. Exploring the role and diversity of mucins in heath and disease with special insight into non-communicable diseases. Glyconj J 2015; DOI 10.1007/s10719-015-9606-6.

25. Li Y, Zhou C, Li J, Llu J, Lin L, Li L, Cao D, Li Q, Wang Z. Single domain based bispecific antibody, Muc1-B1, and its humanized form, Muc1-B2, induce potent cancer cell killing in mucl positive tumor cells. PLoS ONE 2018; 13: e0191024. http://doi.org/10.1371/journal.pone.0191024.

26. Paulsen F, Langer G, Hoffman W, Berry M. Human lacrimal gland mucins. Cell Tissue Res 2004; 316: 167177. 2004

27. Hamada T, Matsukita S, Goto M, Kitajima S, Batra SK, Irimura T, Sueyoshi K, Sugihara K, Yonezawa S. Mucin expression in pleomorphic adenoma of salivary gland: a potential role for MUC1 as a marker to predict recurrence. J Clin Pathol 2004; 57: 813-821.

28. Gao P, Zhou GY, Song XR, Hou JX, Zhang CJ, Ma C. The relationship of abnormal expression of cell glucoprotein with recurrence of pleomorphic adenoma in salivary gland. Hua Xi Kou Qiang Yi Xue Za Zhi 2005; 23: 164-166.

29. Zhang P. Tang LJ, Gao HH, Zhang WX, Lin JX, Yang HS. Immunohistochemical features of carcinoma ex pleomorphic adenoma and pleomorphic adenoma in the lacrimal gland. Int J Ophthalmol 2019; 12: 1238-1242.

30. Ma S, An F, Li H, Lin YY, Wang J. Expression of Mucin 1 in salivary gland tumors and its correlation with 
clinicopathological factors. J Biol Regul Homeost Agents 2019; 33: 563-569.

Corresponding to /correspondencia a: Dra. María Elena Samar Universidad Nacional de Córdoba, Facultad de Odontología Haya de La Torre s/n Ciudad Universitaria CP5000 $T e:+543515146432$

Email/Correo electrónico: maria.elena.samar@unc.edu.ar 